\title{
Fully Integrated Electrothermal Multidomain Modeling of RF MEMS Switches
}

\author{
Brian D. Jensen, Kazuhiro Saitou, Member, IEEE, John L. Volakis, Fellow, IEEE, and \\ Katsuo Kurabayashi, Member, IEEE
}

\begin{abstract}
RF MEMS switches have demonstrated excellent performance. However, before such switches can be fully implemented, they must demonstrate high reliability and robust power-handling capability. Numerical simulation is a vital part of design to meet these goals. This paper demonstrates a fully integrated electrothermal model of an RF MEMS switch which solves for RF current and switch temperature. The results show that the beam temperature increases with either higher input power or increased frequency. The simulation data are used to predict switch failure due to temperature-related creep and self pull-in over a wide range of operating frequency $(0.1-40 \mathrm{GHz})$ and power input $(0-10 \mathrm{~W})$. Self pull-in is found to be the dominant failure mechanism for an example geometry.
\end{abstract}

Index Terms-Power handling capability, RF MEMS.

\section{INTRODUCTION}

$\mathbf{E}$ MERGING and future autonomous, wireless communications systems require highly reliable electronic components with very low power consumption. Micromechanical switches present a promising technology to meet this demand. Such RF switches have been demonstrated with low loss, low power consumption, low distortion, and higher off-state isolation as compared to $\mathrm{p}$-n diodes or field effect transistors [1]-[4]. However, before such switches can be used in commercial applications, they must demonstrate the ability to switch reliably over billions of cycles. The high cost of packaging such devices has also limited their commercial acceptance. Moreover, their power handling capability is normally much lower than $1 \mathrm{~W}$, and reliability concerns become more pronounced for higher power [5]. Careful modeling would allow design of more reliable switches with better power handling capability.

Many of the possible failure mechanisms for MEMS switches, such as electromigration, creep, adhesion, buckling, self pull-in, and surface degradation, are related to the thermal behavior of the switch. Even small electrical losses can cause significant temperature rise in microscopic parts, making thermal modeling essential to understand and predict failure [6], [7]. At high frequencies, the electron crowding caused by the skin effect and by edge effects for rectangular

\footnotetext{
Manuscript received November 18, 2002; revised March 31, 2003. This work was supported by the National Science Foundation under Grant ECS-0115222 and by the Central Intelligence Agency under Contract 2001-H605400-000. The review of this letter was arranged by Associate Editor Dr. Rüdiger Vahldieck.

The authors are with the University of Michigan, Ann Arbor, MI 48109-2122 (volakis@umich.edu).

Digital Object Identifier 10.1109/LMWC.2003.817121
}

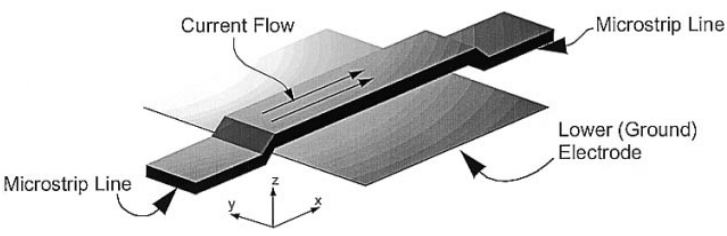

Fig. 1. RF MEMS switch schematic (not drawn to scale).

conductors tends to make this problem worse. Hence, simultaneous modeling of both electrical and thermal effects is needed to better understand switch failure. Previous work in electrothermal modeling includes simulation of temperature rise due to dc current flow [8] and low-frequency magnetic induction heating [9], [10]. This paper links high-frequency electromagnetic simulation and steady-state thermal modeling, providing multidomain solutions. We present sample model data to demonstrate trends, and we use the data to evaluate two possible failure modes.

\section{INTEGRATED ELECTROTHERMAL MODELING}

Fig. 1 shows a simplified view of a RF MEMS switch which consists of a current-carrying beam. To short the switch to ground, electrostatic force is applied to pull the beam down to contact a portion of the lower electrode. This type of switch can be implemented, for example, in a microstrip line, with the beam acting as a portion of the line and the lower electrode attached electrically to the ground plane. Note that this switch design differs from those analyzed in [6] and [7] because the beam carries all of the transmission line current while in the up state. In the previously analyzed designs, a beam was suspended above a CPW line, and the beam carried only a small current in the up state.

\section{A. Electric Current Modeling}

For this work, the beam is modeled in the up state shown in Fig. 1. With RF current flowing through the beam, the average heat generated is equivalent to the electrical power loss, given by

$$
p_{\text {loss }}=\frac{\|\mathbf{J}\|^{2}}{2} \rho
$$

where $p_{\text {loss }}$ is the average power loss per unit volume, $\mathbf{J}$ is the current density within the beam, and $\rho$ is the electrical resistivity. Hence, to calculate the heat generated in the beam, we must first evaluate the current density for the beam geometry, frequency, and electrical boundary conditions. It is well known that at high frequencies, self-inductance of the conductor causes 


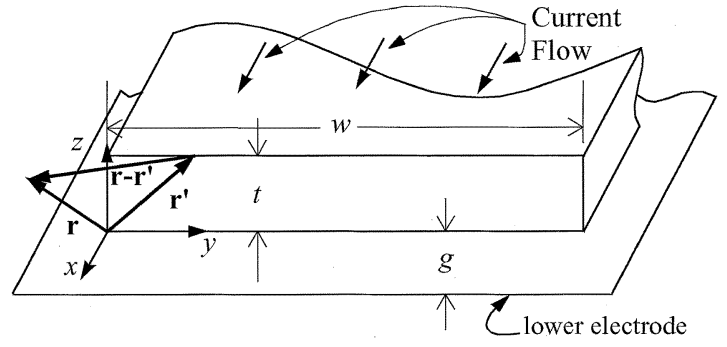

Fig. 2. Beam cross section used in electromagnetic modeling.

electrons to crowd toward the outside edges of the beam, with increased current crowding at higher frequency. As a result, large current density values exist near the beam edges which follow the form $\mathbf{J} \sim 1 / \sqrt{y}$, where $y$ is the distance from the edge of the beam. Further, the skin depth at RF frequencies is on the same order as the beam thickness. Thus, a current density solution within the beam is required. While analytical solutions exist for the current density on wires with a circular cross section, numerical modeling is required for a rectangular conductor.

A full-wave, three-dimensional numerical simulation is likely to give the most accurate results. However, three assumptions enhance the simplicity of the model.

- The electric field in the beam remains substantially parallel to the beam's length (the $x$-direction in Fig. 1). Thus, any $y$ - or $z$-components of the current can be ignored.

- The field variation along the $x$-direction is very small. Consequently, it may be modeled only in the $y-z$ plane.

- The effects of capacitance between the lower electrode and the beam in the up state are small, and are ignored.

These assumptions allow 2-D modeling on a transverse beam cross section normal to the $x$-axis, as illustrated in Fig. 2.

The fields on the cross section are modeled using the finite element-boundary integral (FE-BI) method [11], [12]. Within the beam, the electric field must satisfy the wave equation

$$
\nabla^{2} E_{x}-\gamma^{2} E_{x}=0
$$

where $\gamma$ is the complex propagation constant in the metal. This equation is solved via the finite element method. The fields outside and on the boundary of the beam can be expressed using Kirchhoff's integral equation as

$$
\begin{aligned}
E_{x}(\mathbf{r})=-\oint\left\{G\left(\mathbf{r}, \mathbf{r}^{\prime}\right) \frac{\partial}{\partial n^{\prime}}(\right. & \left.E_{x}\left(\mathbf{r}^{\prime}\right)\right) \\
& \left.\quad-E_{x}\left(\mathbf{r}^{\prime}\right) \frac{\partial}{\partial n^{\prime}} G\left(\mathbf{r}, \mathbf{r}^{\prime}\right)\right\} d l^{\prime}
\end{aligned}
$$

where, as usual, the vector $\mathbf{r}$ refers to the testing point, defining a point outside or on the beam boundary. The vector $\mathbf{r}^{\prime}$ refers to the integration point. Further, $G\left(\mathbf{r}, \mathbf{r}^{\prime}\right)$ is the free space Green's function. Alternatively, the presence of the lower electrode is approximated by using the half-space Green's function. In the context of FE-BI, (2) and (3) are discretized and solved simultaneously to yield the electric field within the beam and on its surface [11], [12]. The current is then obtained from $\mathbf{J}=\sigma \mathbf{E}$, where $\sigma$ is the metal conductivity.

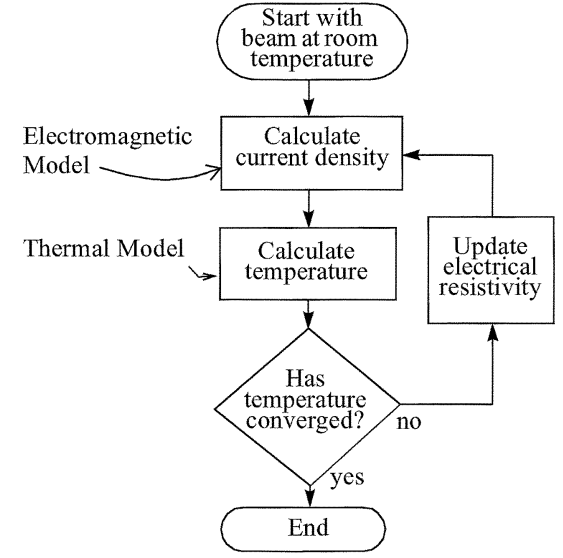

Fig. 3. Flowchart for modeling temperature rise in the switch.

\section{B. Thermal Modeling}

Once the current density is known, (1) is used to find $p_{\text {loss }}$, which is treated as a constant (time-invarying) heat generation. The temperature in the beam is obtained from the steady-state heat equation

$$
\nabla(k \nabla T)=-p_{\text {loss }}
$$

where $\kappa$ is the thermal conductivity and $T$ is the temperature. Because the heat generation term $p_{\text {loss }}$ is evaluated numerically, (4) is also most easily solved with numerical techniques. As with the electromagnetic fields, (4) was solved using the finite element method. Because the thickness of micromachined beams is considerably less than their width, we assumed constant temperature through the beam thickness, so that a 2-D FEM simulation could be performed within the $x-y$ plane. Further, because heat transfer due to conduction is expected to be significantly greater than heat lost due to convection or radiation, only conduction is modeled.

\section{Integrated Modeling}

Equations (1) and (4) describe the temperature dependence on the electrical properties of the beam. Moreover, the electrical resistivity $\rho$ is expected to vary linearly with temperature, and this has been demonstrated experimentally [13]. Consequently, modeling requires iteration to allow temperature changes to update the value of $\rho$. Fig. 3 illustrates a modeling scheme using the relaxation method. Starting with room temperature $\left(25^{\circ} \mathrm{C}\right)$, and a corresponding resistivity, the latter is updated as the temperature changes until convergence is reached. While this iteration scheme is quite simple, it yields satisfactory results. After coding the models in Matlab, we found that two to three iterations were typically required for convergence to within $1 \%$.

\section{FAILURE Mechanisms}

The integrated model can be used to predict switch failure. In this work, we consider creep and self pull-in failure.

Creep Failure: Creep is the time-dependent plastic deformation of materials at elevated temperature even when subjected to stress well below the yield strength. Many materials, including metals, exhibit creep at temperatures above half their melting point (see [14, pp. 215-217]). Hence, a gold switch, having a 


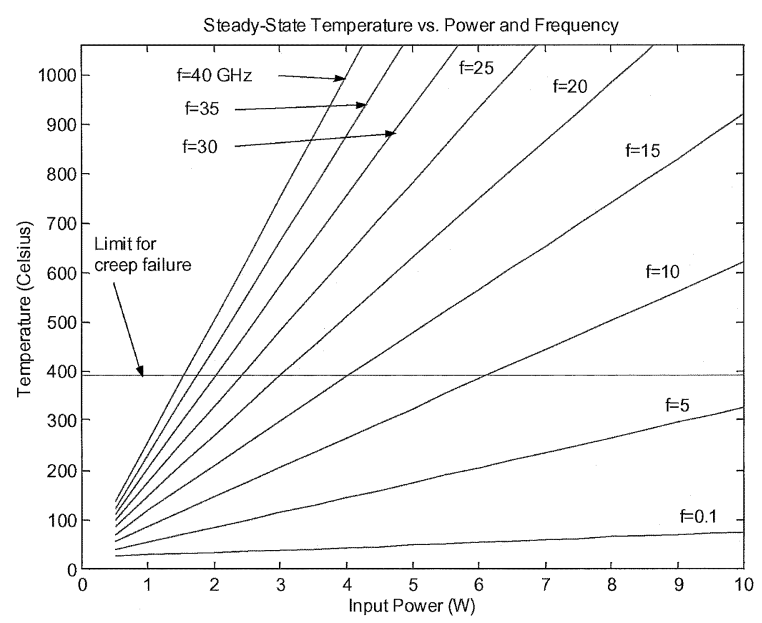

Fig. 4. Maximum steady-state temperature in a beam as a function of power and frequency.

melting point of $1340 \mathrm{~K}$, is likely to fail over time if the temperature exceeds $670 \mathrm{~K}$, or $392^{\circ} \mathrm{C}$.

Self Pull-in Failure: Self pull-in occurs when the signal voltage carried by the switch generates sufficient electrostatic force to pull the beam down, shorting the line. This failure mode depends strongly on temperature because compressive thermal strains generated at elevated temperature drastically reduce the pull-in voltage. The pull-in voltage for a fixed-fixed beam has been previously modeled as a function of internal strain [15]. With this model, we are able to evaluate the pull-in voltage as a function of beam temperature using the total strain $\varepsilon_{T}$ in the beam, given by

$$
\varepsilon_{T}=\varepsilon_{R}+\varepsilon_{t h}=\varepsilon_{R}-\frac{1}{l} \int_{0}^{l} \alpha \Delta T d x
$$

where $\varepsilon_{R}$ is the residual (or initial) strain, $\varepsilon_{t h}$ is the thermal strain, $\alpha$ is the thermal expansion coefficient, and $\Delta T$ is the temperature change. For gold, we used $\alpha=13.8 \times 10^{-6} /{ }^{\circ} \mathrm{C}$ and $\varepsilon_{R}=0.0012$ (tensile), as well as Young's modulus $E=$ $36.0 \mathrm{GPa}$ and Poisson's ratio $\nu=0.4$. The latter three values were reported in [16] for electroplated gold films.

\section{RESULTS AND DISCUSSION}

Fig. 4 shows the maximum steady-state temperature as a function of power and frequency for a gold $400 \times 50 \times 2 \mu \mathrm{m}$ beam suspended $2 \mu \mathrm{m}$ above the ground plane. The temperature scale has been truncated at $1063{ }^{\circ} \mathrm{C}$, the melting point of gold. The limit for expected creep is also marked at $392{ }^{\circ} \mathrm{C}$. The temperature varies practically linearly with input power; similarly, the temperature also increases with frequency. The latter increase is due to the skin effect and the edge effects, and has not been previously modeled.

Using the model data, the two failure modes studied here-creep and self pull-in - can be evaluated, as shown in Fig. 5. For this geometry, self pull-in is the dominant failure mechanism for all frequencies and power levels. A key conclusion of this study is that failure occurs at much lower input power as frequency increases-with pull-in occurring when $P_{\text {in }} \approx 6.3 \mathrm{~W}$ at $f=3 \mathrm{GHz}$, but at about $2.3 \mathrm{~W}$ at $f=10 \mathrm{GHz}$ for this example. Previous modeling efforts

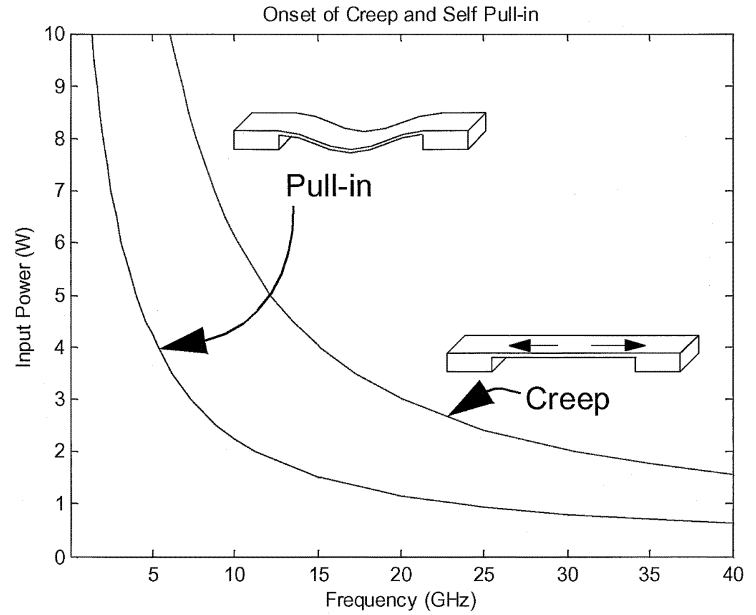

Fig. 5. Predicted onset of self pull-in and creep.

have concentrated on the effect of power [6], but this study for the first time shows that RF MEMS failure is also strongly related to frequency. Moreover, it points out the importance of integrated electrothermal modeling.

\section{REFERENCES}

[1] C. Chang and C. Peizen, "Innovative micromachined microwave switch with very low insertion loss," Sensors and Actuators A: Physical, vol. 79 , pp. 71-75, 2000.

[2] J. B. Muldavin and G. M. Rebeiz, "High-isolation CPW MEMS shunt switches-Part 2: Design," IEEE Trans. Microwave Theory and Tech., vol. 48, no. 6, pp. 1053-1056, June 2000.

[3] J. Y. Park, H. K. Geun, K. W. Chung, and J. U. Bu, "Electroplated RF MEMS capacitive switches," in Proc. of the IEEE Micro Electro Mechanical Systems (MEMS), 2000, pp. 639-644.

[4] J. B. Muldavin and G. M. Rebeiz, "High-isolation CPW MEMS shunt switches-Part 1: Modeling," IEEE Trans. Microwave Theory and Tech., vol. 48, no. 6, pp. 1045-1052, June 2000.

[5] G. M. Rebeiz and J. B. Muldavin, "RF MEMS switches and switch circuits," IEEE Microwave Mag., vol. 2, no. 4, pp. 59-71, Dec. 2001.

[6] J. B. Rizk, E. Chaiban, and G. M. Rebeiz, "Steady state thermal analysis and high-power reliability considerations of RF MEMS capacitive switches," in 2002 IEEE MTT-S Symposium Digest, vol. 1, pp. 239-243.

[7] W. Thiel, K. Tornquist, R. Reano, and L. P. B. Katehi, "A study of thermal effects in RF-MEMS-switches using a time domain approach," in 2002 IEEE MTT-S Symposium Digest, vol. 1, pp. 235-238.

[8] J. Funk, J. G. Korvink, M. Bächtold, J. Bühler, and H. Baltes, "Coupled 3D thermo-electro-mechanical simulations of microactuators," in Proc. IEEE Micro Electro Mechanical Systems (MEMS), 1996, pp. 133-138.

[9] L. Gong, R. Hagel, and R. Unbehauen, "On the 3-D inhomogeneous induction heating of a shell," IEEE Trans. Magnetics, vol. 30, no. 5, pp. 3324-3327, Sept. 1994.

[10] J. Driesen, R. Belmans, and K. Hameyer, "Adaptive relaxation algorithms for thermo-electromagnetic FEM problems," IEEE Trans. Magnetics, vol. 35, no. 3, pp. 1622-1625, May 1999.

[11] J. L. Volakis, A. Chatterjee, and L. C. Kempel, Finite Element Method of Electromagnetics. New York: IEEE Press, 1998.

[12] J.-J. Jin, J. L. Volakis, and J. D. Collins, "A finite-element-Boundaryintegral method for scattering and radiation by two- and three-dimensional structures," IEEE Antennas and Propagation Magazine, vol. 33, no. 3, pp. 22-32, June 1991.

[13] P. G. Borden, "A novel method for measuring the thermal conductivity of electrodeposited gold films," J. Electrochemical Society, vol. 127, no. 11, pp. 2454-2457, Nov. 1980.

[14] C. R. Barret, W. D. Nix, and A. S. Tetelman, The Principles of Engineering Materials. Englewood Cliffs, New Jersey: Prentice-Hall, Inc, 1973.

[15] P. M. Osterberg and S. D. Senturia, "M-TEST: A test chip for mems material property measurement using electrostatically actuated test structures," J. MEMS, vol. 6, no. 2, pp. 107-118, June 1997.

[16] C.-W. Beak, Y.-K. Kim, and Y.-M. Ahn, "Mechanical property measurement of electroplated gold microstructure using resonance method," in MRS Symp. Proc., vol. 605, 2000, pp. 229-234. 